\title{
Strates
}

STRATES Matériaux pour la recherche en sciences sociales

14 | 2008

Espaces du quotidien

\section{Les concepteurs de la ville en quête de l'espace familier (1945-1975)}

\section{Frédéric Pousin}

\section{(2) OpenEdition}

1 Journals

\section{Édition électronique}

URL : http://journals.openedition.org/strates/6722

DOI : 10.4000/strates.6722

ISSN : $1777-5442$

\section{Éditeur}

Laboratoire Ladyss

\section{Édition imprimée}

Date de publication : 1 janvier 2008

Pagination : 191-211

ISBN : 0768-8067

ISSN : 0768-8067

\section{Référence électronique}

Frédéric Pousin, «Les concepteurs de la ville en quête de l'espace familier (1945-1975) », Strates [En ligne], 14 | 2008, mis en ligne le 13 mars 2013, consulté le 08 septembre 2020. URL : http://

journals.openedition.org/strates/6722 ; DOI : https://doi.org/10.4000/strates.6722

Ce document a été généré automatiquement le 8 septembre 2020.

Tous droits réservés 


\title{
Les concepteurs de la ville en quête de l'espace familier (1945-1975)
}

\author{
Frédéric Pousin
}

1 Les concepteurs qui ont à projeter le paysage urbain, qu'ils soient architectes, urbanistes ou paysagistes, manipulent des représentations des territorialités quotidiennes. Et, de fait, la problématique du quotidien, des différents espaces qui lui sont liés, ainsi que leur impact sur la conception de l'architecture, de la ville, ou plus généralement, de l'environnement, s'exprime, expressément ou subrepticement, dans les discours professionnels. Pour décrypter les représentations des territorialités quotidiennes manipulées par les concepteurs, la connaissance de la culture professionnelle, y compris dans sa formation et ses mutations, fournit des éléments de compréhension appréciables.

2 Il s'agira dans cet article d'en analyser la "généalogie ", de mettre en évidence plusieurs gestes qui sont au cœur des pratiques contemporaines d'analyse de l'environnement et d'élaboration d'outils pour la conception.

3 À quels moments, dans quelles circonstances et en relation avec quels enjeux cette problématique a-t-elle joué un rôle ? Dans quelle mesure a-t-elle pu être structurante? Quels en furent les effets? C'est à cet ensemble de questions que nous tenterons d'apporter des éléments de réponse. Nous nous pencherons plus particulièrement sur les outils mis en œuvre par les professionnels pour donner corps à cette problématique, c'est-à-dire sur les représentations qui permettent à la fois de saisir ces « espaces du quotidien", mais aussi de les manipuler, de les "projeter» dans des opérations d'aménagement. Car l'hypothèse que nous faisons consiste à lier fondamentalement cette problématique des territoires du quotidien aux dispositifs mis en œuvre pour les rendre visibles, manipulables, et, plus particulièrement à deux dispositifs de représentation: l'enquête photographique d'une part, la notation du mouvement de l'autre. Notre corpus, sélectionne des discours centrés sur la représentation. Nous nous attacherons à suivre les effets en aval de ce qui s'est constitué à un moment historique donné. 


\section{La critique de la modernité architecturale après la seconde guerre mondiale : Townscape et Team 10}

4 Dans les domaines de l'architecture et de l'urbanisme, l'intérêt pour les pratiques spatiales au quotidien est concomitant à la remise en cause du modèle de la ville fonctionnelle. Une telle remise en cause prend des formes diverses selon les différentes cultures nationales. Elle s'exprime de manière précoce et avec force en Angleterre suivant deux directions qui se déclarent concurrentes mais qui, de fait, poursuivent un objectif commun: énoncer de nouvelles valeurs pour la modernité. Une première direction cherche à fonder ces nouvelles valeurs à partir d'un ancrage national. L'esthétique pittoresque, alors considérée comme un idiome britannique, sera revisitée pour inspirer une vision de l'urbanisme, le townscape, qui intègre la dimension du paysage afin de dépasser l'opposition ville-campagne, d'accorder toute sa place à la notion de site ainsi qu'à la perception. L'autre direction tentera de dégager de nouvelles bases pour une modernité qui, non seulement reconduit la revendication internationale des Congrès internationaux d'architecture moderne (CIAM) ${ }^{1}$, mais de plus élargit les référents aux cultures vernaculaires des pays colonisés.

La critique des valeurs modernistes qui s'énonce alors peut apparaître à certains égards paradoxale, car elle s'appuie sur un double mouvement, d'élargissement et de rétrécissement du point de vue. Un geste d'ouverture qui consiste à élargir le point de vue de l'urbanisme vers un « art de l'environnement » intégrateur ou vers de nouveaux référents culturels, en même temps qu'un geste de focalisation sur les pratiques et les espaces du quotidien. La dimension paradoxale que l'on peut percevoir ici se lève en partie si l'on considère que la critique du modernisme s'élabore à partir de ses frontières, de ses marges. Toutefois, il conviendra de garder présent à l'esprit le contrepoint de la focalisation qui s'opère sur le quotidien.

6 Dès les premières formulations du townscape (1949), la perception y joue un rôle central et le le visuel prédomine (optics). Il s'agit d'une perception dynamique : l'espace urbain ne peut être pensé indépendamment d'un sujet qui le perçoit et qui est mobile. La prise en considération du mouvement, à l'échelle du piéton en particulier, constituait un enjeu. 
Figure 1. Gordon Cullen, Townscape, 1961, p. 18-19.

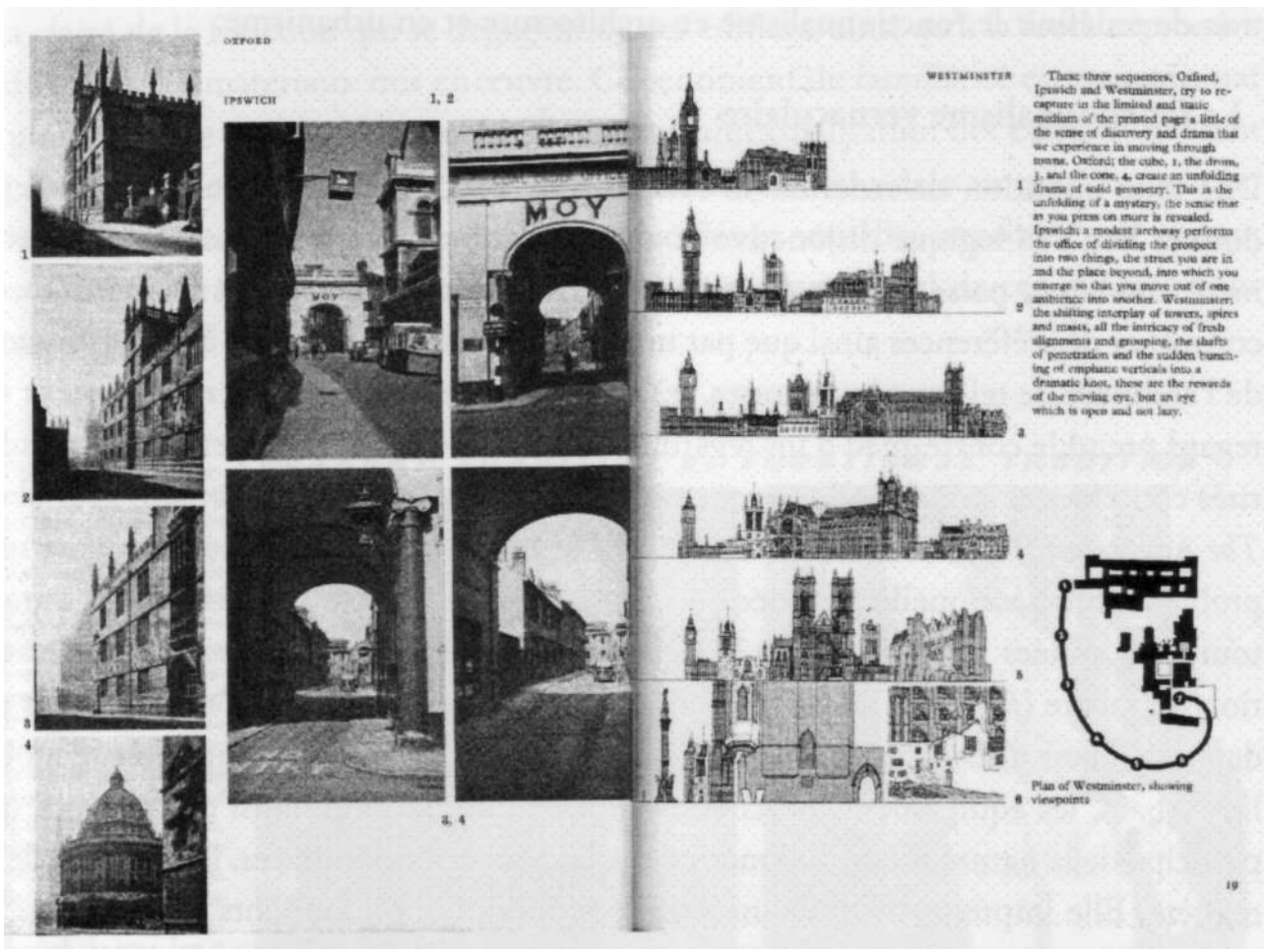

L'optique expliquée à travers la catégorie de vision sérielle. Trois séquences : Oxford, Ipswitch, Westminster.

Reproduced courtesy of The Architectural Review.

7 En 1949, dans le numéro 636 de la revue londonienne The Architectural Review, Gordon Cullen publie un Townscape Casebook (recueil d'exemples symptomatiques) censé établir les bases d'un «art de l'environnement » à partir d'une typologie des manières de voir objectivées (Cullen, 1949). Parmi les différents types de vision proposés, l'un d'entre eux se construit explicitement sur le mouvement, l'œil y étant assimilé à une caméra de cinéma (eye as a movie-camera). L'exemple convoqué, un parcours autour de Westminster Abbey, sera repris dans le livre de synthèse publié en 1961, Townscape (Cullen, 1961), pour établir la catégorie essentielle de vision sérielle, témoignant du rôle majeur joué par la dynamique visuelle chez les architectes anglais se réclamant du townscape $e^{2}$ (figure 1). Pour eux, l'appréhension de la ville, du cadre urbain, est fondamentalement liée au déplacement et fonctionne suivant des séquences. La perception en mouvement renvoie alors à une pratique ordinaire de la ville. Cette pratique ordinaire, qui se traduit par des usages différents d'un même espace à différents moments de la journée ou en fonction des jours, s'exprime dans la catégorie de multiple use (usage multiple), catégorie antonyme de ségrégation.

En même temps que se met en place une approche de l'urbanisme élargie à l'environnement perçu et pratiqué, la critique des valeurs modernistes passe par l'intention de redéfinir le fonctionnalisme en architecture et en urbanisme.

\section{Le fonctionnalisme vernaculaire}

9 Une telle intention de redéfinir la catégorie du fonctionnalisme, tel qu'il avait pu donner lieu à la logique disjonctive et abstraite du zoning appliquée par le mouvement 
moderne, passe par un élargissement du domaine des objets susceptibles de constituer des références ainsi que par une focalisation du regard sur des constituants de l'architecture tels que les textures, les matériaux, les couleurs. Ce resserrement du regard prend le contre-pied d'un regard planificateur qui s'attache peu aux particularités concrètes et se confond souvent avec une vue de l'esprit. Un numéro spécial de The Architectural Review de 1950, consacré à la tradition fonctionnelle, inaugure une problématique à laquelle sera dédiée une rubrique et des articles isolés, ce durant toutes les années 1950. Il définit la tradition fonctionnelle en opposition à la tradition populaire (figure 2). La première s'incarnerait dans les ouvrages tels les jetées ou dans les objets utilisés dans la marine, les signaux routiers, les inscriptions, le mobilier urbain, les équipements du chemin de fer et des rivières, ainsi que dans ce qui participe de la nature même des matériaux, les textures des surfaces, le caractère de la matière. Elle imprègne le domaine urbain et rural. Pour les tenants du townscape, la qualité d'un paysage urbain est dépendante de la qualité de ses détails. Avant d'engager la réflexion sur le paysage urbain, un préalable consiste, aux yeux des rédacteurs de la revue, à collecter, analyser, amener à la conscience de tous les éléments et objets de la scène urbaine anonyme, banals et fortuits, ne faisant l'objet d'aucune reconnaissance particulière, ni, pour certains, d'une identification visuelle: pavements, emmarchements, garde-corps, etc. Le dossier incite le lecteur à prêter attention et à analyser des objets relevant d'un environnement national familier, qui recèlent de nombreuses qualités pour les projets d'aménagement. Collectés grâce à la photo, ce sont des objets déjà là (Lichtenstein, 2001) au sens où il s'en dégage des qualités plastiques et poétiques non intentionnelles. Les projets d'aménagement illustrés par les dessins de G. Cullen, montrent des espaces de loisirs pratiqués dans la quotidienneté de leur usage, sans mise en scène exceptionnelle.

Outre la rubrique qui a pourvu cette thématique d'un foisonnement d'exemples et de discussions, The Architectural Review consacre, en juillet 1957, un nouveau numéro spécial à " The Functional Tradition as seen in Early Industrial Buildings ${ }^{4}$ ». Signé par James Maud Richards et composé des photographies d'Éric de Maré, ce numéro est consacré aux moulins, aux docks, aux entrepôts, aux brasseries qui constituent l'architecture industrielle des XVIII ${ }^{e}$ et XIX ${ }^{e}$ siècles. Le quotidien y est toutefois peu présent, si ce n'est grâce au sentiment de familiarité lié à la simplicité de la forme et à la clarté de la fonction qui se dégagent de ces édifices, ainsi qu'à la force expressive qui émane des matériaux mis en œuvre. Ce sentiment de familiarité est transmis par la photographie qui s'attache au détail ou à la contextualisation des édifices, mettant l'accent sur leur statut d'objets déjà là. Leur rassemblement dans un numéro met en valeur la puissance des qualités formelles, tactiles et visuelles exprimée par chacun des clichés; il révèle en tout cas le caractère non intentionnel de ces qualités pour chacun des édifices en particulier. 
Figure 2. The Functional Tradition, The Architectural Review, 1950, vol. 107, n 637, p. 6.

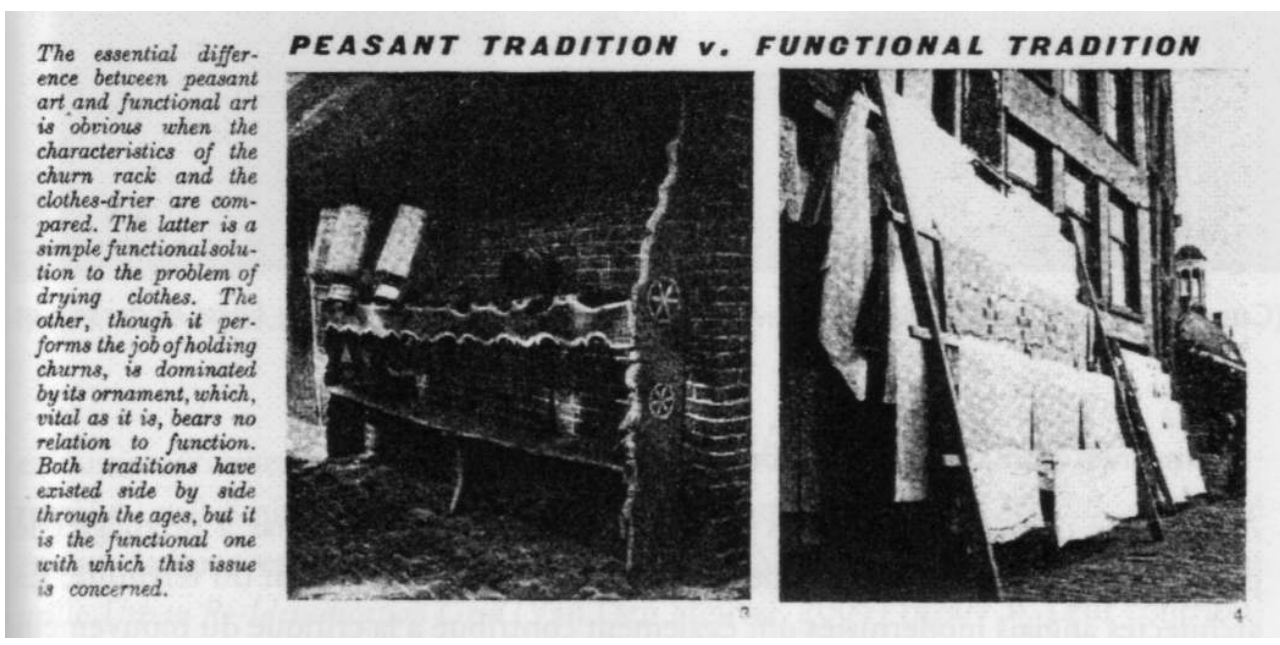

Tradition fonctionnelle vs tradition paysanne.

Reproduced courtesy of The Architectural Review.

11 Nous ne nous attarderons pas plus sur ce numéro, dont l'intérêt pour une histoire de la notion de fonctionnalisme en architecture dépasse l'objet de cet article (Higgott, 1990).

La réinvention du fonctionnalisme suppose, on le voit, un resserrement du regard sur les objets matériels de la quotidienneté, resserrement que sert parfaitement la photographie, tout comme elle sert la valorisation du point de vue du piéton. Autant de caractéristiques qui représentent des alternatives à l'abstraction des chiffres et des schémas qui sont utilisés alors couramment dans la planification.

\section{Un nouvel humanisme : les valeurs de la sociabilité}

À partir de positions doctrinales différentes, qui ne cherchent pas à privilégier le paysage, mais à placer au c œur des préoccupations l'appropriation du territoire, les architectes anglais modernistes ont également contribué à la critique du mouvement moderne dans l'après-guerre. Alison et Peter Smithson jouèrent un rôle décisif dans l'émergence d'attitudes nouvelles au sein de l'équipe anglaise participant aux CIAM, le groupe MARS ${ }^{5}$. Celles-ci s'expriment lors du IX congrès des CIAM, en 1953, consacré à la discussion de la charte de l'habitat (Pédret, 2005). La notion d'habitat apparaissait problématique, car très polysémique, différant peu en l'espèce des termes de logis ou logement débattus auparavant. Avec une différence notable toutefois, l'habitat permettant de référer plus largement à l'environnement, notion supposée capable de conjoindre les dimensions spirituelles et physiques propres aux habitants. Lors du IX congrès des CIAM, la jeune génération revendique de penser la ville en termes d'habitat, se démarquant ainsi des architectes restés fidèles aux idées de la charte d'Athènes. Cette génération se reconnaissait dans un désir commun de créer des environnements capables d'encourager les relations entre les habitants, entre les bâtiments et leurs environnements et qui puissent s'accommoder aux besoins culturels des habitants. A. et P. Smithson y présentent leur diagramme Hierarchy of Association préparé en association avec leurs collègues du groupe MARS, Bill et Gill Howell, ainsi que leur grille Urban Re-Identification Grid (Van Den Heuvel, 2005) (figure 3). Leur contribution consistait à remplacer la hiérarchie fonctionnelle «logis, travail, 
circulation, loisir » par des catégories plus phénoménales et entretenant une relation scalaire, telles que maison, rue, district (district), et ville, recouvrant ainsi des niveaux différents de « rassemblement humain ».

Figure 3. Grille pour le CIAM IX (Congrès international d'architecture moderne), 1953 ; photographies de Nigel Anderson et diagrammes d'Alison et Peter Smithson.
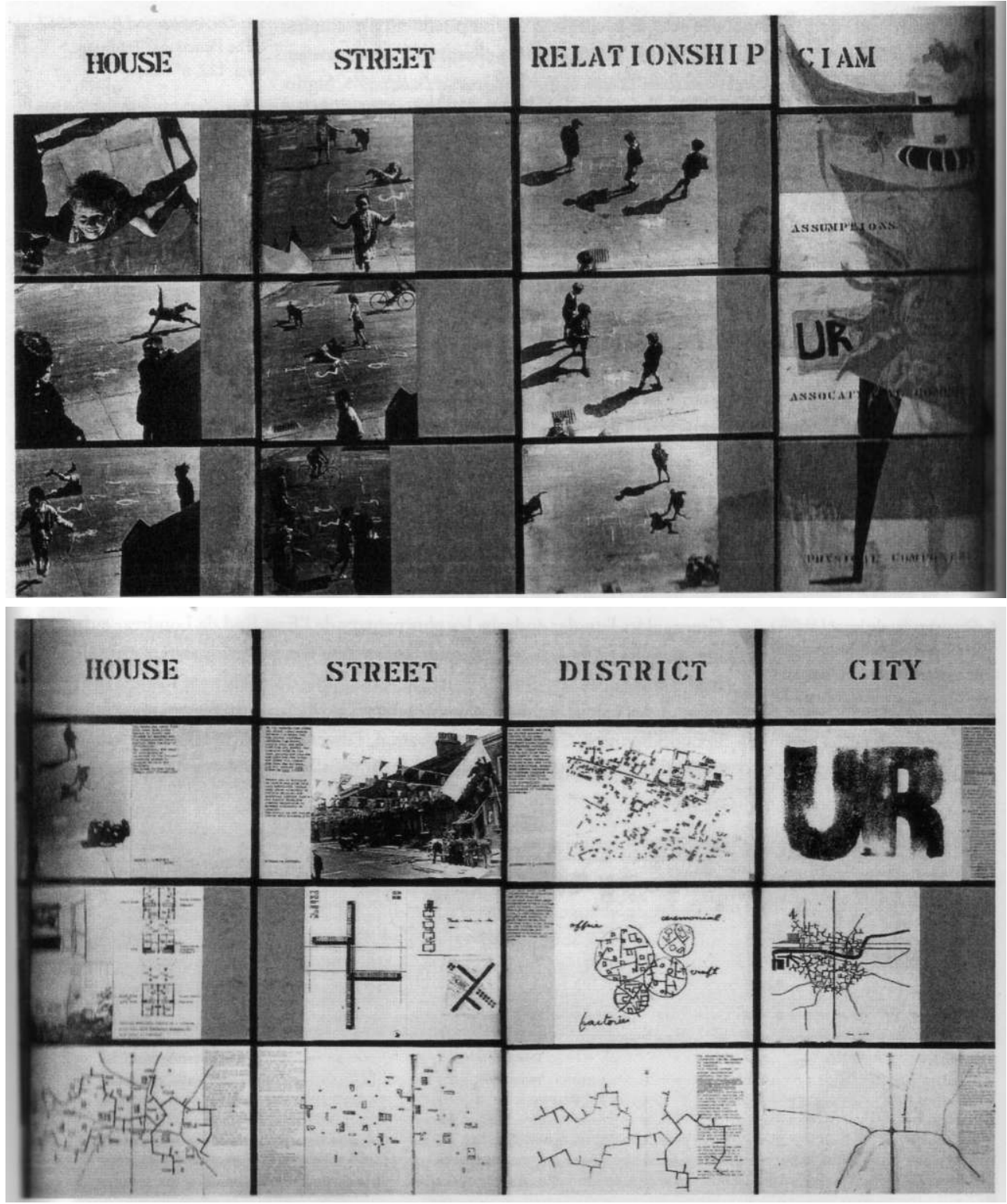

(c) DR. A+P Smithson Archives.

14 La partie droite de la grille présentée par A. et P. Smithson reprend des idées issues de leur projet pour Golden Lane Estate à Londres. La rue, qui constitue un concept clé de leur projet, est déconnectée de son contexte urbain et combinée à des blocs de logements formant une superstructure. Les « corridors ", ou rues intérieures de l'unité d'habitation de Le Corbusier, sont rejetés en façade, exhibant les vicissitudes de la vie quotidienne.

15 La partie gauche inclut des photos de Nigel Henderson, un artiste de l'Independant Group. Ce sont des images d'enfants jouant dans les rues de Bethnal Green, alors l'un des 
endroits les plus pauvres de l'East End de Londres : enfants faisant de la bicyclette, du patin à roulettes, sautant à la corde, jouant à la marelle... Ces jeux d'enfant condensent et illustrent un ensemble de valeurs qui inspirèrent le mouvement des jeunes architectes des derniers CIAM, Team $10^{6}$ et qui nous ramènent à la quotidienneté. Ce sont l'improvisation, l'invention, la chorégraphie urbaine, une sociabilité impromptue, la flexibilité territoriale, etc.

L'image d'enfants jouant sur le pas de la porte constituait l'emblème d'un nouvel humanisme porté par un certain nombre d'écrits sur la crise de la ville occidentale contemporaine ${ }^{7}$. Ainsi, au milieu des années 1950, les ethnographes Michael Young et Peter Willmott réalisent une étude de Bethnal Green visant à évaluer les schémas du changement social dans la vie de la classe ouvrière. Les résultats furent publiés en 1957 sous la forme d'un livre Family and Kinship in East London (Young, Willmott, 1957), qui fut très important pour lutter contre les effets négatifs des opérations de résorption des taudis. Le transfert des populations ouvrières dans les complexes du nouveau logement de masse s'accompagnait d'un démantèlement des communautés ouvrières traditionnelles et des formes de sociabilités qu'elles avaient pu générer. L'ouvrage décrivait la sociabilité familiale comme un réseau étendu de parents et de familiers vivant en contiguité, de proches, sans lien de sang particulier, qui contribuaient à la prise en charge des enfants, aux relations d'amitiés, aux liens sociaux. Dans les années 1950, une telle image de la "famille de la rue » apparaissait en danger. L'accent mis sur cet aspect de la vie "familiale » quotidienne constituait une mise en garde contre de nouvelles formes d'habitat et d'environnement qui risquaient de balayer des marques fondamentales de la sociabilité humaine. Les images de ces enfants jouant dans les rues de l'East End n'en étaient pas moins ambivalentes, soulignant autant les capacités humaines à construire à partir de rien que l'extrême fragilité d'un monde social au bord de l'effondrement.

17 Family and Kinship, comme la Urban Re-Identification Grid, valorisaient la localité, l'association humaine immédiate, et les formes sociales de spontanéité.

Aux valeurs défendues par les CIAM précédents - la piazza italienne emblème du c œur de la cité ou une nouvelle monumentalité - les jeunes architectes modernistes opposaient les rues du East london ouvrier comme potentiel d'inspiration pour de nouvelles formes de vie en architecture et urbanisme. Le terme de re-identification - qui donna lieu à un article désormais célèbre d'A. et P. Smithson, paru dans Architectural Design en $1955^{8}$ - exprimait l'idée que les formes architecturales et urbaines à venir reposaient sur des valeurs nouvelles auxquelles la population doit pouvoir s'identifier.

Lors du congrès des CIAM IX, le projet d'A. et P. Smithson ne souleva pas un intérêt très grand, contrairement à la valeur d'icône qu'il acquit par la suite. En revanche, certains projets, qui prenaient en considération les conditions locales, discutaient les valeurs spirituelles, repensaient le statut de l'habitat dans les pays colonisés, retinrent l'attention des participants (Çelik, 2005b). Ainsi des projets du groupe marocain GAMMA $^{9}$ et du CIAM Alger, pour la reconstruction du bidonville de Mahiedine (Çelik, 2005a) (figure 4), dans lesquels les espaces des couches les plus pauvres des pays colonisés se révélaient porteurs d'inspiration. 
Figure 4. Extrait de la grille « Bidonville Mahieddine » 1953, grille présentée au CIAM IX.

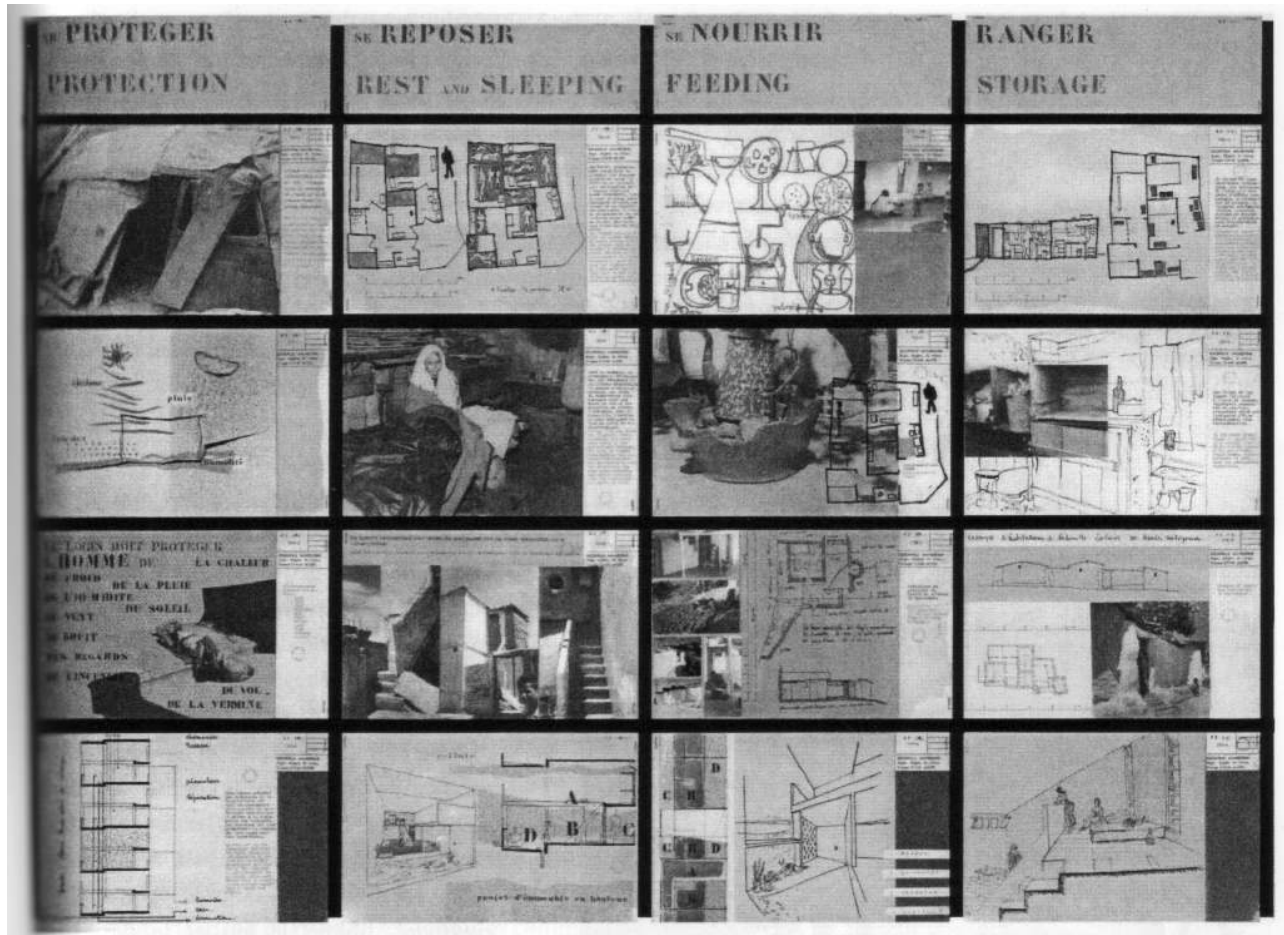

(C) DR. NAI Publishers, Rotterdam.

Dans l'étude approfondie du bidonville de Mahiedine, le regard porté sur les formes d'organisation spatiales en révélait la dignité. Il s'agissait pour l'équipe des architectes et urbanistes d'exprimer un savoir qui puisse être utile à la communauté des CIAM tout entière. Le propos, franchement orienté sur les habitants et les formes construites du bidonville, mettait en œuvre une méthodologie multidisciplinaire, et les informations contenues dans la grille étaient portées par diverses formes d'expressions visuelles, tout comme dans le projet du groupe d'architectes modernes marocains, intitulé « Habitat du plus grand nombre ", qui présentait un projet de logements à Casablanca, Carrières centrales, réalisé par l'Atelier des bâtisseurs (ATBAT) sous la houlette de Georges Candilis et des services de l'urbanisme dirigés par Michel Écochard. 
Figure 5. Aldo van Eyck, aires de jeux pour enfants à Amsterdam.

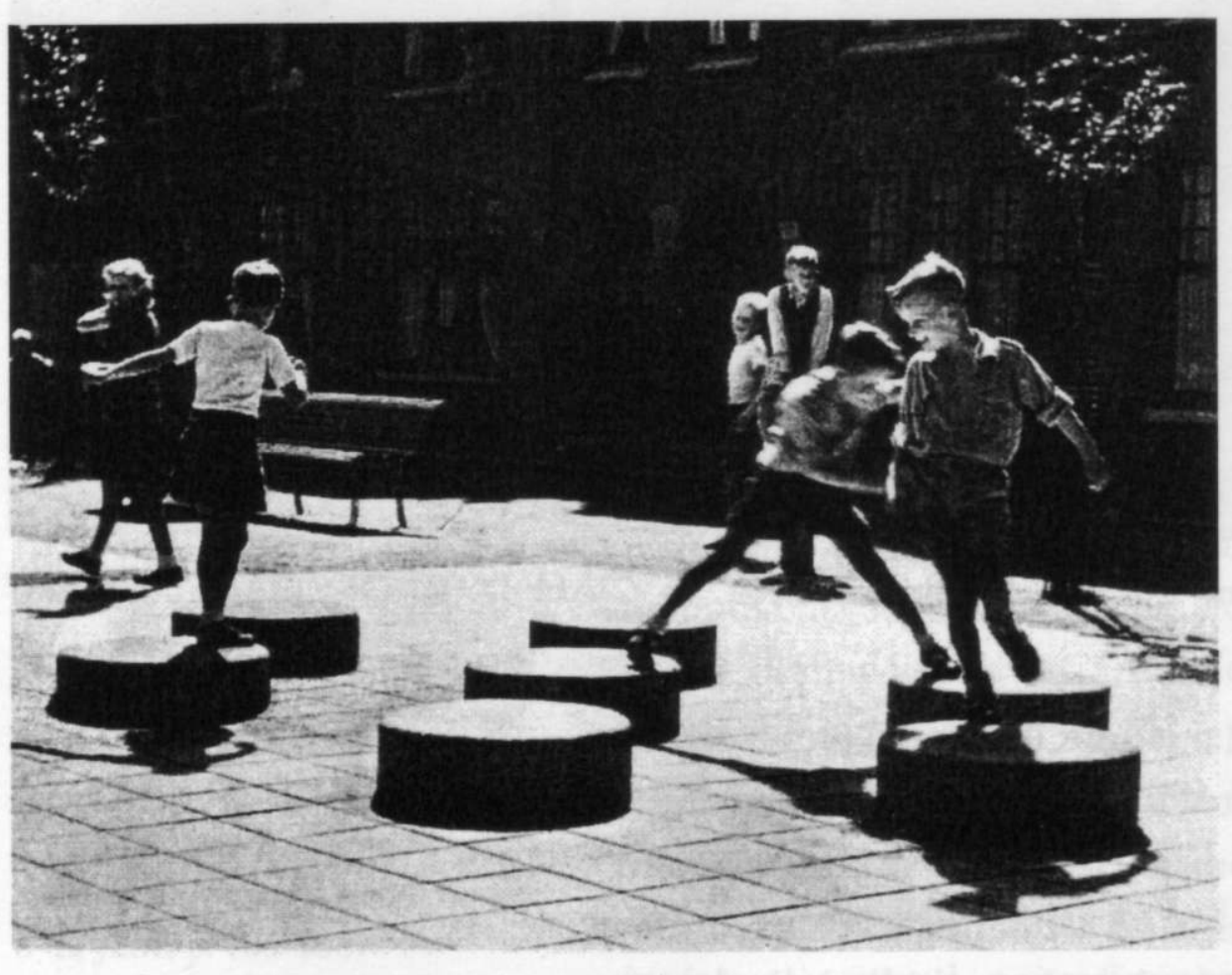

(c) DR. NAI Publishers, Rotterdam.

21 Si le sujet du logement de masse était dans le droit fil des CIAM, la manière de le traiter était tout à fait originale. L'approche se voulait intégrale et qualitative à la fois. Comme chez A. et P. Smithson, l'orientation qualitative était fondée sur une analyse des environnements construits du quotidien. Alors qu'A. et P. Smithson trouvaient leur inspiration dans une analyse photographique de la classe laborieuse d'une rue de Londres, le point de départ du groupe Gamma était la vie quotidienne et le bâti dans un bidonville. Un atelier ambulant, joignant des compétences diverses (ingénieur, topographe, urbaniste, dessinateurs) et s'appuyant sur des travaux sociologiques et anthropologiques préalables décrivaient conjointement les types d'habitat et les modes de vie, grâce à des relevés photographiques (Eleb, 2000). Traiter du banal et de la réalité quotidienne était un choix délibéré pour Sadrach Woods car « le spectaculaire n'est que de peu d'intérêt dans le domaine de l'habitat et de l'urbanisme ${ }^{10}$ ».

\section{Le quotidien saisi par la photographie}

Le Team 10 comptait parmi ses membres les plus en vue, aux côtés d'A. et P. Smithson, G. Candilis et S. Woods. Faisant écho au structuralisme naissant, le Team 10, dans sa recherche de l'appréhension du comportement humain, balançait entre l'idéalisation de l'attitude spontanée, et l'affirmation de règles collectives partagées. Le parcours d'Aldo van Eyck est à cet égard représentatif. Il a été à la recherche d'une enfance de l'architecture comme les surréalistes ont été en quête de l'enfance de l'écriture, ou comme les membres de Cobra ont utilisé les dessins d'enfants pour interroger la créativité, libérer le jeu et le désir. Cela explique l'attention qu'il a portée à la réalisation d'aires de jeux pour enfants à Amsterdam. La représentation 
photographique qu'il en donne se rapproche d'ailleurs de celle d'A. et P. Smithson à travers le choix qu'ils font des clichés de N. Henderson (figure 5).

L'intérêt pour le quotidien en photographie trouve aussi l'une de ses origines dans l'engouement ethnographique et anthropologique qui caractérise les années 1930. Il convient d'évoquer l'épisode britannique Mass Observation, peut-être moins connu que d'autres entreprises photographiques liées à la crise, mais impliquant une dimension anthropologique comparable à celle d'Auguste Sander en Allemagne (Lugon, 2001) ou celles de la Farm Security Administration (FSA) aux États-Unis. Mass Observation (Mellor, 2005) prenait place, en Grande-Bretagne, dans le contexte des effets de la grande crise économique de 1929. Le monde ouvrier, fortement paupérisé apparaissait comme un monde à part, mal connu, pour lequel la recherche d'images positives du travail et du citoyen constituaient une composante populiste du genre documentaire alors très en vogue à la charnière des années 1940. Ce mouvement, né de l'intérêt d'un éditeur, Victor Gollancz, pour un projet d'ouvrage documentaire de Tom Harrison, l'un de ses trois principaux fondateurs ${ }^{11}$, participe d'une vogue du reportage et du documentaire, portée par les facteurs caractéristiques de la période: une forme de populisme, le développement des études statistiques de la société, l'intérêt pour l'ethnologie et l'anthropologie, et dans le domaine artistique, le surréalisme.

Mass Observation constituait une entreprise hybride qui amalgamait non seulement le sondage d'opinion, l'étude de marché ainsi que l'anthropologie à un moment où l'anthropologie de soi était très porteuse (Winkin, 1981). Au moment où T. Harrison effectuait une enquête sociologique empirique à partir de son activité de camionneur à Bolton fin 1936, il rencontra un groupe d'intellectuels surréalistes qui s'intéressaient à la manière dont l'opinion publique rencontrait les phobies ou les désirs qui s'exprimaient autour des événements faisant les titres de l'actualité comme l'incendie du Cristal Palace ou l'abdication d'Édouard VIII. C'est à l'occasion de cette rencontre que l'entreprise Mass Observation ${ }^{12}$ fut fondée et fit appel à des volontaires et des observateurs pour réaliser des reportages sur leur vie quotidienne, leur travail, leur désirs et leur rêves.

Faire des listes ou collecter des données sur la culture de masse quotidienne constitua une grande part du génie de l'« inspection sociale » à laquelle se sont livrés les acteurs de Mass Observation. La collecte des données fut perçue par Charles Madge, Tom Harrison et Humphrey Jennings comme un premier pas vers un modèle d'observation scientifique qui devait se combiner avec une technique impersonnelle, objective, de l'enregistrement permise par la photographie, grâce à l'appareil photographique 24x36. Il faut voir là aussi un écho aux recherches théoriques de Marcel Duchamp dans le domaine de l'art ou aux écrivains qui pratiquaient l'écriture «clinique ». Le "fait» pour les acteurs de Mass Observation n'était pas sans être empreint d'une aura surréaliste, dont la catégorie du "merveilleux» pouvait être atteinte dans la photographie urbaine (figure 6). H. Jennings était dans l'Angleterre des années 1930 un artiste d'avant-garde qui entretenait des liens d'amitié avec André Breton, Louis Aragon et René Magritte. 
Figure 6. Mass Observation, Blackpool, Amusement park out of season, 1936.

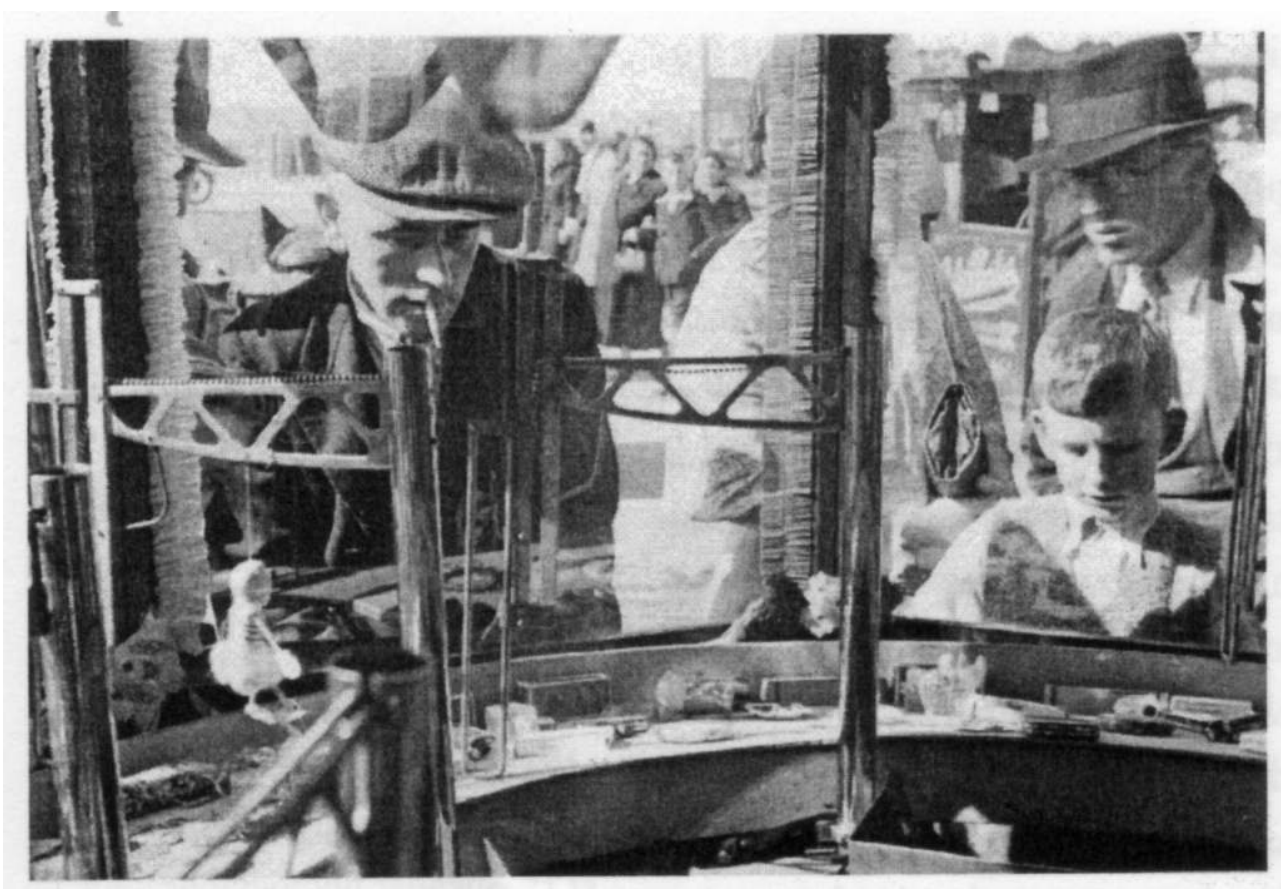

(c) DR. La fabrica Editorial.

26 Ainsi l'enquête ethnographique portée par la photographie a-t-elle été, pour toute une génération d'architectes et d'urbanistes, à l'origine d'un renouveau du regard porté sur la ville et l'habitat, dans leur relation à l'environnement. Au c œur de ce renouveau se trouve la description des pratiques ordinaires du quotidien, leur signification anthropologique aussi bien que leurs valeurs au sein de formes de sociabilités situées culturellement. On l'a vu, les formes de représentation au moyen desquelles sont figurées ces pratiques, et grâce auxquelles elles circulent, ne sont pas anodines.

Le souci pour la mobilité qui, dès le début des années 1950, s'était exprimé comme une autre composante essentielle des valeurs constitutives d'une relation renouvelée à la ville, donnera lieu, dans les années 1960, à des développement originaux, à partir d'une réflexion sur la créativité et d'une interrogation sur la relation corporelle à l'espace. Au centre du débat se trouve la question de la notation, en tant que possibilité de représentation et de manipulation du mouvement. Dans les recherches et pratiques contemporaines, on le verra plus loin, la dimension de la mobilité reste un enjeu important pour comprendre et concevoir les espaces qui configurent le quotidien.

\section{La saisie du mouvement}

C'est aux États-Unis, où le contexte s'y prête tout particulièrement, que la réflexion investit le terrain de la notation du mouvement en tant que système symbolique au fondement d'une pratique créative.

Philippe Thiel développa un travail significatif et original dans ce domaine, tout au long des années 1950. L'article qu'il publia en 1961 (Thiel, 1961) synthétise un travail initié en $1951 \mathrm{au}$ Massachusetts Institut of Technology (MIT), lors d'un cours de diplôme dédié au réaménagement visuel d'une section de la ville de Boston. Il s'agissait de concevoir un cheminement piéton reliant différents points historiques de la ville. Ce 
diplôme a été l'occasion de penser les relations séquentielles entre éléments et de prendre conscience qu'il n'existait pas de moyen satisfaisant de mesurer, non plus que de représenter sous forme de schémas, une telle expérience. S'en est suivie une recherche de dix ans, bénéficiant des apports de Gyorgy Kepes, principal collaborateur de Moholy Nagy au New Bauhaus de Chicago, puis enseignant au MIT et de Kevin Lynch qui y développait ses travaux sur la forme perceptive de la ville, recherche financée par le College of Architecture de l'université de Berkeley. L'hypothèse de départ, reprise au mouvement anglais du townscape est que la perception visuelle de notre environnement est un processus dynamique incluant le temps.

Figure 7. Deux tableaux tirés de Philippe Thiel, «A sequence -experience notation » @ Reproduced courtesy of The Townplanning Review, vol. XXXII, nº1, avril 1961, p. 42.

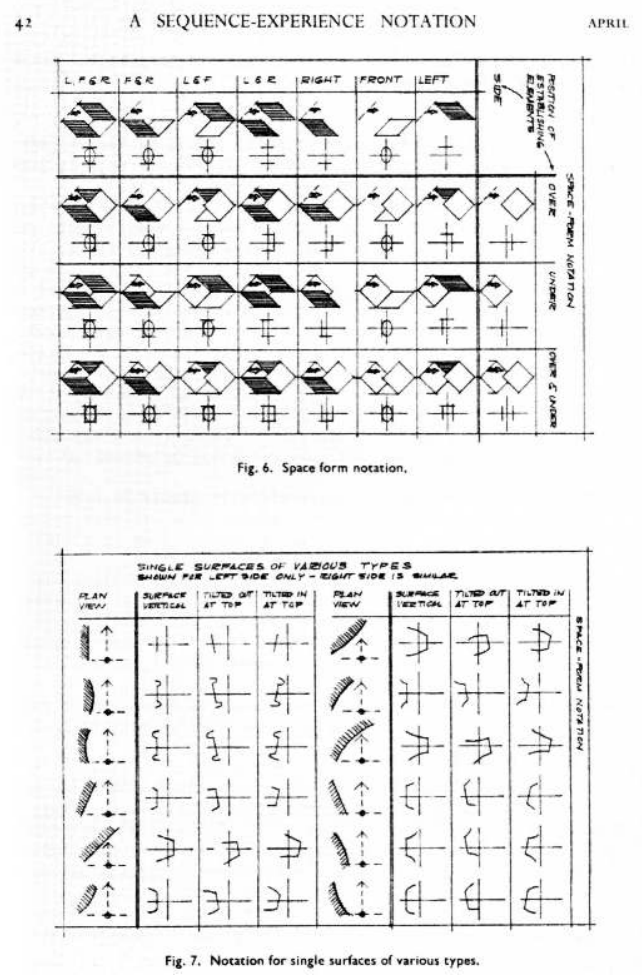

En haut : notation pour des formes dans l'espace. En bas : notation pour des formes de différents types.

Pour P. Thiel, le mouvement dans le temps est une préoccupation constante du musicien, du danseur et du réalisateur et qui tendait à devenir un sujet de préoccupation pour les architectes, urbanistes, planificateurs urbains et paysagistes, sous l'effet du changement d'échelle de l'aménagement et de l'expérience des paysages en mouvement, grâce à la pratique massive du voyage. Si le musicien dispose d'une partition, le danseur de la notation Laban, le réalisateur d'un story-board, les architectes et designers ne disposaient jusqu'alors que d'outils de représentation statiques. L'objectif de $\mathrm{P}$. Thiel consista alors à mettre au point un système graphique de notation pour représenter l'expérience des séquences architecturales et urbaines.

31 Son système de notation est construit en trois étapes: la première vise l'anatomie de l'espace architectural et urbain, la seconde, les sens, la signification de l'expérience spatiale, la troisième articule les deux premières par le mouvement. En référence à la musique et à la chorégraphie, la ligne du mouvement est construite sur une référence 
temporelle. La ligne est à lire verticalement, avec les intervalles de temps d'un côté de la ligne, la distance de l'autre (figure 7).

Les travaux de P. Thiel furent développés par D. Appleyard, K. Lynch et J.-R. Meyer (Appleyard et al., 1964) et appliqués au déplacement automobile, à la perception depuis la route, sans pour autant s'intéresser à l'usage au quotidien de l'automobile, raison pour laquelle nous ne nous étendrons pas sur ce point.

\section{Notation du mouvement, conception, participation : Lawrence Halprin}

La notation de l'expérience séquentielle du corps en mouvement connut des développements, dans le champ de l'architecture et du paysage, développement qui orientèrent la réflexion vers la dimension procédurale de l'activité de conception ainsi que vers la diversité des activités ordinaires, effectuées au quotidien. C'est Lawrence Halprin qui fut amené à explorer les potentiels de la notation, qu'il dénomma score terme que l'on traduira par partition ou notation - à des fins de conception participative.

Très tôt, L. Halprin s'est intéressé au mouvement corporel, aux capacités à se mouvoir, au sens du déplacement. Son goût et sa curiosité pour la danse, la proximité de son épouse Ann, impliquée dans les recherches avant-gardistes en danse contemporaine l'ont conduit à observer et interroger ce qui procure la sensation du mouvement, le libère. "Le mouvement humain, particulièrement au théâtre et en danse, grâce au travail conjoint avec mon épouse, la danseuse Ann Halprin, m'a conduit vers des recherches sur les manières de montrer le mouvement et de concevoir en fonction de celui-ci, ce que j'ai nommé Motation... Plus tard, cela a conduit à des explorations de processus sans fin et de la notion de notation, notamment la compréhension de son rôle clé dans la manière dont on arrive à des solutions » (1971, p. 10). 


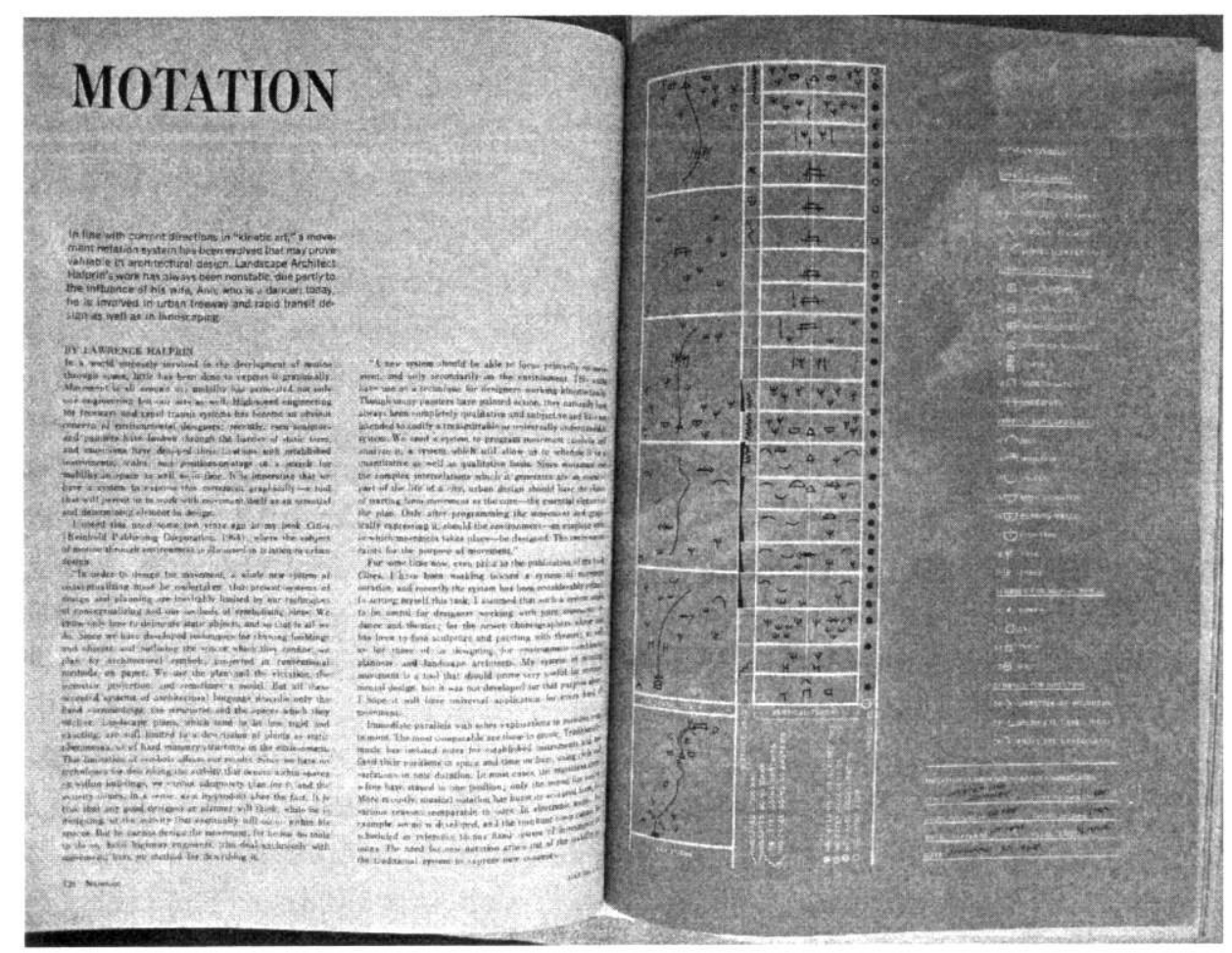

(c) DR

Dans les années 1965, L. Halprin met au point un système de notation du mouvement en gestation depuis plusieurs années - qu'il dénomme «motation» et qui permet d'évaluer et de concevoir les environnements humains en fonction du mouvement du corps. L. Halprin partage avec les architectes qui se sont penchés sur le sujet, le souci de mettre à disposition des concepteurs, un système de représentation cohérent et simple qui permette autant de programmer le mouvement que de l'analyser, de le schématiser sur une base à la fois quantitative et qualitative. L'article qu'il publie dans la revue Progressive Architecture en 1965, intitulé "Motation» (notation du mouvement) (Halprin, 1965), positionne clairement sa démarche dans la lignée de l'art cinétique, tout en soulignant les implications de sa recherche pour la conception architecturale et le design environnemental (figure 8). Il puise dans ce domaine d'application des traits caractéristiques telles que les données plurisensorielles qui sont notées de manière combinée avec la distance.

Dans les années 1966-1968, à l'heure du mouvement hippie et de la contestation politique, L. Halprin développe des «expérimentations environnementales » dans le cadre d'ateliers pluridisciplinaires, où sont conduites des expériences de relations interpersonnelles prises dans leur cadre environnemental. Fortement orientées sur l'observation des processus à l'œuvre, ces expériences ont débouché sur une approche générale de la créativité qui a donné lieu à la fois à une "philosophie " de l'action créative et à un livre The RSVP Cycles (Halprin, 1970). RSVP désignent les termes d'un cycle qui permet de rendre les choses visibles, de décrire et de travailler avec des processus visant des objectifs plutôt que des buts prédéterminés. 


\section{La partition : un élément matriciel}

37 Au cœur des expérimentations se trouve l'idée de "partition », qui est pensée comme une matrice organisatrice pour toute sorte d'activités, allant du planning quotidien aux événements incarnant la fantaisie et le cérémoniel, en cohérence avec un intérêt pour l'anthropologie très répandu alors (Winkin, 1981). Elle est pensée comme un dispositif permettant l'appropriation individuelle, dans l'activité quotidienne, d'un cadre réglé et partagé. Les partitions sont des indications d'actions humaines dans le temps et dans l'espace. Une partition ne se réduit pas nécessairement à une notation, elle désigne plus largement toute forme de représentation qui combine espace, temps et activité pour pouvoir donner lieu à une mise en acte, sur le mode de la performance. Par exemple, un plan de site ou d'édifice constitueraient une expression basique de la partition qui, mis en actes, produirait un nouvel environnement. Le nouvel environnement pourrait, luimême, être une partition tridimensionnelle dans la manière dont il met en forme le mode de vie au quotidien (travail et loisir).

L'ouverture ou la fermeture d'une partition ont été un vaste sujet de réflexion pour L. Halprin $^{13}$.

39 La partition ouverte est celle qui laisse le plus d'initiative à l'interprète, par conséquent elle contient peu d'instructions, voire d'indications, l'interprétation se générant à travers son procès. La partition fermée est celle qui comprend un maximum de déterminations que l'interprète est invité à suivre. Aux yeux de L. Halprin, la partition ouverte n'est pas supérieure sur la partition fermée, elles correspondent seulement à des types de situations différentes, renvoyant à des cadres plus ou moins réglés. Il s'agit de savoir comment une partition aide les «interprètes » à mieux répondre à une situation.

\section{Partition, créativité et participation}

À la fin des années 1960 et dans les années 1970, les partitions ouvertes servent des objectifs d'épanouissement personnel et de créativité, ancrés dans une pensée processuelle omniprésente. Ainsi, des partitions ouvertes consacrées à l'élargissement de la perception des processus et de la créativité chez les individus ont été mises en œuvre dans une série d'ateliers comme celui pour le National Endowment for the Arts (Fonds national pour les arts), visant à susciter, chez les technocrates fédéraux, une prise de conscience du processus de conception.

41 L'apport des ateliers et des partitions est allé bien au-delà du seul développement de la créativité. L'enseignement des années 1966-1968 a permis d'ancrer la conviction suivant laquelle la conscience de la communauté est déterminante pour son évolution. Les ateliers visent à générer des formes de familiarisation et d'appropriation. Dès lors, L. Halprin s'est fortement impliqué pour introduire dans la planification urbaine les techniques de partition ouvertes et de participation fondée sur le cycle RSVP. Les actions conduites suivant ces principes de motivation des acteurs, d'interaction personnelle, de scénarii alternatifs, et d'appropriation collective ont produit des résultats notables en termes d'environnement bâti. Les espaces concernés ne sont pas exceptionnels, ce sont ceux de la vie au quotidien, les espaces du travail, du loisir, des multiples activités que la ville accueille. Ainsi, à Fort Worth, au Texas, en 1969, ces actions ont donné lieu à la formulation d'un plan directeur pour le quartier d'affaire du 
centre puis en 1976 à la réhabilitation d'un système de parcs le long de la boucle de la Trinity River qui traverse Fort Worth. L'expérience des ateliers expérimentaux a nourri également des opérations de réhabilitation des centre-villes en déclin comme à Charlottesville (Virginia), victime de l'expansion de la banlieue et des centres commerciaux. Invité en 1973 à mettre en place des ateliers de planification municipale avec les habitants, les commerçants et les représentants de la mairie pour établir des plans de réhabilitation du centre-ville, Halprin et Associates a réalisé ensuite l'aménagement piétonnier de l'artère du centre-ville, achevé en 1975. Le processus d'implication des acteurs dans la prise en charge de leur propre environnement en fonction de leurs activités au quotidien a été dénommé « prendre part (take part) » et a donné lieu à un ouvrage publié en 1972 (Halprin \& Associates, 1972). Les phénomènes à l'œuvre de Familiarisation, d'appropriation et de prise en charge s'enchaînent.

Dans les ateliers mettant en œuvre partition et participation, les concepteurs agissent comme les chefs d'orchestre du processus. Ils favorisent la créativité, le mouvement et aident à se détourner des situations de confrontation, des rassemblements publics, ou des décisions par vote qui produisent immanquablement un groupe de perdants. En ce sens, les processus participatifs proposés par L. Halprin, renouvellent, aux États-Unis, la tradition d'une planification portée par le débat public (advocacy planning). "Les mécanismes d' "écoute active" en tant que moyen de communication opposé à l'exposition de points de vue. Cela a entraîné loin du plaidoyer vers le Cycle RSVP ${ }^{14}$... » L'appropriation est le maître mot des partitions à des fins de conception et des processus de participation.

L. Halprin a exploré dans ses méandres, la vertu de mise en commun dont une forme de notation est porteuse, sa capacité à impliquer les gens dans des activités partagées, à travers performances et créativité. Les partitions seraient autant des guides que des activateurs.

Aujourd'hui se développent des méthodes scientifiques d'analyse et de caractérisation des ambiances de la ville (au Cresson ${ }^{15}$ notamment), permettant d'avoir une bonne appréciation des qualités d'ambiance pour concevoir les lieux de vie urbaine. L'observation de l'usage de l'espace, du déplacement des corps, de ses divers modes d'appropriation par les piétons qui cheminent, révèle une vie sociale, une dynamique urbaine ainsi que des qualités d'ambiance spécifiques à chaque moment de la journée et à chaque lieu de la ville, autant d'espaces et de temporalités de la vie quotidienne. Des méthodes d'investigation sont basées sur l'observation in situ de scènes ou de situations urbaines appartenant à différents lieux de la ville et à divers moments de séquence de temps. Ces méthodes visent à révéler des interactions qui sont déterminantes entre, d'une part les comportements et les usages, d'autre part, les facteurs spatiaux et les conditions microclimatiques. La notion de pattern ambiant qui résulte de ces analyses organise les différentes composantes spatiales, temporelles, climatiques, comportementales. Mais surtout, la notion de pattern s'accompagne d'une visée opératoire au sein des opérations, voire des phases de la conception des espaces urbains. En effet, les patterns sont des figures élaborée à partir d'une typologie des scènes urbaines et des modes d'appropriation, et de leur combinatoire. 


\section{Conclusion}

Notre enquête sur les formes de représentation du quotidien produites et manipulées par les concepteurs, architectes, urbanistes et paysagistes, montre que l'intérêt pour cette problématique a trouvé un nouveau souffle au lendemain de la seconde guerre mondiale et qu'il est lié à la volonté d'ancrer la pensée sur l'humain dans un nouveau terreau. Rappelons que dans le domaine de la philosophie, la première version de la Critique de la vie quotidienne d'Henri Lefebvre est parue en 1947 (Lefebvre, 1947). Bien sûr, le quotidien n'est pas une thématique inédite, elle est au cœur de plusieurs démarches dès les années 1930, notamment les surréalistes. Ainsi nous avons pu suivre en Grande-Bretagne le fil qui relie Mass Observation aux mouvements lancés par les architectes anglais depuis la fin des années 1940. L'usage ethnographique de la photographie s'avère être un fil rouge convaincant. Mais alors que les surréalistes ont été amenés à étudier des moments exceptionnels comme les fêtes ou les grands événements, à la manière dont les anthropologues se penchaient sur le rituel, ici ce sont les moments ordinaires, répétitifs, constituant l'environnement familier que l'on cherche à décrire, et dont la pensée de l'architecture et de la ville est sommée de s'imprégner. De ce point de vue, nous pouvons considérer que les processus de familiarisation (Bégout, 2005), pour reprendre l'expression de Bruce Bégout, sont au centre de l'intérêt pour le quotidien et sa spatialisation. L'espace est appréhendé comme un cadre pour un corps qui est tout sauf statique. Le mouvement, le déplacement supposent, en outre, la prise en considération du temps. La recherche sur le mouvement et son indexation temporelle s'est développée particulièrement OutreAtlantique. Elle a débouché sur la question de l'appropriation qui a été pensée en termes de créativité, à la faveur des bouleversements culturels des années 1965-1970. Elle diffère du concept marxiste d'appropriation auquel H. Lefebvre a eu recours. Chez L. Halprin, l'appropriation se construit sur les relations interpersonnelles, y compris lorsque l'appropriation est appréhendée à l'échelle de la collectivité. Ainsi, la familiarisation relevant de l'appropriation se fonde sur une forme d'intersubjectivité, cet article est ainsi à même d'éclairer une réflexion plus ample sur la notion de quotidien, apportant des éléments de réflexion, du point de vue de la conception, sur les processus de territorialisation - au sens de l'appropriation d'un espace par un sujet qui le transforme en un lieu familier. Elle apporte également des éléments de compréhension de la culture professionnelle, des architectes aux paysagistes. Elle souligne le rôle joué par les intermédiaires (Latour, 1989), dont la photographie, qui articulent l'espace abstrait de la conception sur un espace matériel situé culturellement et historiquement. Enfin, l'éclairage sur le moment historique délimité permet de porter un regard mieux informé sur les travaux contemporains sur les ambiances architecturales et urbaines, qui se dotent de méthodes scientifiquement construites pour explorer minutieusement des problématiques qui ont un passé. 


\section{BIBLIOGRAPHIE}

Appleyard D., Lynch K., Myer J.R., 1964, The View from the Road, Cambridge, Massachusetts, MIT Press.

Avermaete T., 2005, « Habitat du plus grand nombre Grid 1953 », in Risselada M.,Van Den Heuvel D. (eds), Team 10 1953-81, In Search of a Utopia of the Present, Rotterdam, NAI Publishers, p. 26-29.

Bégout B., 2005, La découverte du quotidien, Paris, Allia.

Çelik Z., 2005a, « Bidonville Mahiedine Grid, 1953 », in M. Risselada, D. Van Den Heuvel (eds), Team 10 1953-81, In Search of a Utopia of the Present, Rotterdam, NAI Publishers, p. 22-25.

Çelik Z., 2005b, « The Ordinary and the Third World at CIAM IX », in Risselada M.,Van Den Heuvel D. (eds), Team 10 1953-81, In Search of a Utopia of the Present, Rotterdam, NAI Publishers, p. 276-279.

Eleb M., 2000, « An Alternative to Functional Universalism : Écochard, Candilis and ATBATAfrique », in Goldhagen S.W., Legault R. (eds), Anxious Modernisms, Cambridge and Montreal, MIT Press, p. 363-374.

Cullen G., 1949, « Townscape Casebook », The Architectural Review, vol. 106, n 636. Cullen G., 1961, Townscape, London, Architectural Press. Grosjean M., Thibaud J.-P., 2001, L'espace urbain en méthodes, Marseille, Éditions

Parenthèses.

Halprin L., 1965, « Motation », Progressive Architecture, July, p. 126-133.

Halprin L., 1970, RSVP Cycles : Creative Processes in the Human Environement, New York, Georges Brazillier.

Halprin L., 1971, Notebooks, 1959-1971, Cambridge, Massachusetts and London, England, The MIT Press.

Halprin L. \& Associates, 1972, Take Part, Portland, Oregon, Porland Press.

Higgott A., 1990, Éric de Maré Photographer Builder with Light, London, Architectural Association.

Higmore B., 2005, « Rescuing Optimism from Oblivion », in Risselada M.,Van Den Heuvel D. (eds), Team 10 1953-81, In Search of a Utopia of the Present, Rotterdam, NAI Publishers.

Lefebvre H., 1947, Critique de la vie quotidienne, Paris, L'arche.

Latour B., 1989, La science en action, Paris, La Découverte (traduction française).

Lichtenstein, Cl. Schregenberger (eds), 2001, The Discovery of the Ordinary, Baden, Lars Müller Publishers.

Lugon O., 2001, Le style documentaire, d'August Sander à Walker Evans, 1920-1945, Paris, Macula.

Mellor D. A., 2005, « Mass Observation », Ciudad, PhotoEspana 05 (exhibition book), Madrid, La Fabrica.

Mumford E., 2000, The CIAM Discourse on Urbanism, 1928-1960, Cambridge, MIT Press.

Pédret A., 2005, « CIAM IX : Discussing the Charter of Habitat », in Risselada M.,Van Den Heuvel D. (eds), Team 10 1953-81, In Search of a Utopia of the Present, Rotterdam, NAI Publishers, p. 20-21. 
Pousin F., 2001, « Construire les visualisations du paysage urbain. Pratiques anglaises et américaines de l'après seconde guerre ", Les cahiers de la recherche architecturale et urbaine, $\mathrm{n}^{\circ} 8$, mai, p. 51-61.

Pousin F., 2003, «Visuality as politics. The example of urban landscape », in Dorrian M., Rose R. (eds), Deterritorialisation... Revisioning Landscape and Politics, London, Black Dog Publishing,

p. 161-174.

Thiel P., 1961, « A Sequence-Experience Notation for Architectural and Urban Spaces », The Town Planning Review, $\mathrm{n}^{\circ} 1$, April.

Van Den Heuvel D., 2005, « Urban Re-Identification Grid, 1953, Alison and Peter Smithson », in Risselada M.,Van Den Heuvel D. (eds), Team 10 1953-81, In Search of a Utopia of the Present, Rotterdam, NAI Publishers, p. 30-33.

Winkin Y. (dir.), 1981, La nouvelle communication, Paris, Seuil.

Young M., Willmott P., 1957, Family and Kinship in East London, London, Routledge \& Kegan Paul.

\section{Revues}

Architectural Design, 1955, June, $\mathrm{n}^{\circ} 6$

The Architectural Review, 1950, The Functional Tradition, vol. 107, nº 637.

The Architectural Review, 1957, The Functional Tradition, vol. 122, $\mathrm{n}^{\circ} 726$.

\section{NOTES}

1. Sur les CIAM, voir E. Mumford, 2000.

2. Pour davantage d'informations, voir F. Pousin, 2001 et 2003.

3. The Architectural Review, The Functional Tradition, 1950, vol. 107, $\mathrm{n}^{\circ} 637$.

4. The Architectural Review 1957, The Functional Tradition, vol. 122, $\mathrm{n}^{\circ} 726$.

5. Modern Architectural Research Group, voir E. Mumford, 2000.

6. Groupement de jeunes architectes européens qui se rencontrèrent au travers des CIAM et tentèrent d'établir une nouvelle plate-forme pour l'architecture moderne, différente de celle qui fut largement dominée par Le Corbusier.

7. Les enfants jouant dans l'environnement urbain, inscrivant leur marque dans la ville, représentent un lieu commun du photojournalisme et des enquêtes de sociologie (H. Levitt: photographies d'enfants jouant sur les terrains vagues de New York; E. Smith : photographies d'enfants à Pittsburg, photographie d'enfants jouant dans les réservoirs d'eau à Harlem). Les aires de jeux pour enfants à Londres, Berlin, Rotterdam, Amsterdam,... envahissent les journaux et magazines de l'après-guerre. Voir B. Higmore, 2005.

8. A. et P. Smithson, 1955, « The Built World : Urban Re-Identification », Architectural Design, $\mathrm{n}^{\circ}$ 6, June, p. 185-188.

9. GAMMA, groupe d'architectes modernes marocains qui inclut V. Bodianski, G. Candilis, M. Ecochard, H. Piot et S. Woods,(T. Avermaete, 2005).

10. S. Woods, cité par Z. Çelik, 2005b, p. 277.

11. Avec $C$. Madge, poète et reporter au Daily Mirror et $H$. Spender photographe.

12. L'entreprise eut un certain succès qui se traduisit par un Penguin spécial intitulé «Britain » ainsi qu'à de nombreux articles dans la presse grand public et à des émissions de radio à la BBC. T. Harrison devint rapidement une personnalité radiophonique.

13. Voir « At the Delos symposium. Comments to the group. 16th July 1971 », in L. Halprin, 1971, p. 366-368. 
14. Idem.

15. Centre de recherche sur l'espace sonore et l'environnement urbain, UMR 1563, CNRS, École d'architecture de Grenoble.

\section{RÉSUMÉS}

La problématique du quotidien, des espaces familiers, dans la conception de l'architecture, de la ville et plus largement de l'environnement, s'est manifestée dans les discours professionnels. Il s'agit ici d'en retracer la "généalogie ", et d'analyser les circonstances et les enjeux auxquels cette problématique est liée. Dans quelle mesure a-t-elle pu être structurante et quels en furent les effets? C'est à cet ensemble de questions que cet article s'efforce d'apporter des éléments de réponse en se penchant plus particulièrement sur les outils mis en œuvre par les professionnels, c'est-à-dire sur les représentations (photographies, notations) qui permettent à la fois de saisir ces " espaces du quotidien », dans la diversité de leur appropriation, mais aussi de les manipuler, de les "projeter" dans des opérations d'aménagement. L'hypothèse que nous avons faite consiste à lier fondamentalement cette problématique des territoires du quotidien aux dispositifs mis en œuvre pour les rendre visibles, manipulables.

The issues of everyday life and familiar spaces in architectural conceptions of cities and more generally the environment are now part of professional reflections. Here we propose to establish their "genealogy" and analyse the circumstances and stakes related to the issues posed by everyday life. To what extent could this be a structuring element and what would be the effects? This article tries to answer these questions, particularly by looking into tools used by professionals, for example representations, such as photographs and notations, which allow the depiction of "everyday life spaces" in all the diversity of their accommodation and also their manipulation, for example "projecting" them within development schemes. Our hypothesis consists in establishing a fundamental link between everyday life territories and the way they are made visible and malleable.

\section{INDEX}

Mots-clés : quotidien, architecture, urbanisme, projets urbains, aménagement, épistémologie

\section{AUTEUR}

\section{FRÉDÉRIC POUSIN}

Architecte,

Directeur de recherche, Ladyss (Laboratoire dynamiques sociales et recomposition des espaces), CNRS, Université de Paris 1 ,

2 , rue Valette,

75005 - Paris

frpousin@univ-paris1.fr 